\title{
NORMAL STRUCTURE AND WEAKLY NORMAL STRUCTURE OF ORLICZ SEQUENCE SPACES \\ BY
}

THOMAS LANDES

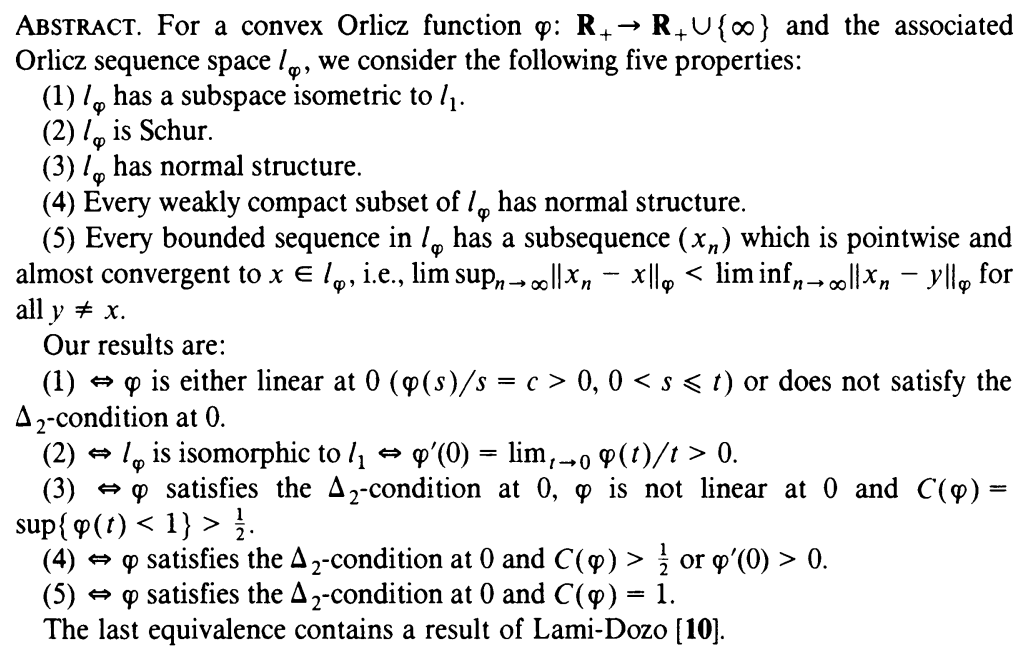

1. Preliminaries. Let $X$ be a normed space and $A$ be a nonvoid subset of $X$. The set $A$ is called diametral if $A$ is bounded and if

$$
\sup \{\|x-y\| \mid y \in A\}=d \text { for all } x \in A \text { and some } d>0 \text {, }
$$

and $A$ is said to have normal structure (cf. [2]) if $A$ has no convex diametral subset.

The normed space $X$ is said to have weakly normal structure if every weakly compact subset of $X$ has normal structure.

Simple examples of sets with normal structure are the subsets of uniformly convex spaces and the compact subsets of any normed space. More examples and information may be found in [12].

Normal structure is one of the fundamental tools in fixed point theory of nonexpansive mappings. We mention only the fixed point theorem of Browder [3], Göhde [6] and Kirk [8] which states that every nonexpansive (i.e., having Lipschitzconstant 1) selfmap of $A$ has a fixed point provided $A$ has normal structure and is weakly compact and convex.

For a given sequence $\left(x_{n}\right)_{n \in \mathrm{N}}$ in $X$, we associate two functionals

$$
\Lambda^{*}(x):=\limsup _{n \rightarrow \infty}\left\|x_{n}-x\right\|, \quad \Lambda_{*}(x):=\liminf _{n \rightarrow \infty}\left\|x_{n}-x\right\|
$$

Received by the editors December 17, 1982.

1980 Mathematics Subject Classification. Primary 46B20, 47H09. 
on $X$ (cf. [10]). If $\left(x_{n}\right)_{n \in \mathbf{N}}$ is bounded, $\left(x_{n}\right)_{n \in \mathbf{N}}$ is called limit-constant (limitaffine) if the two functionals $\Lambda^{*}$ and $\Lambda_{*}$ coincide on the convex hull of $\left\{x_{n} \mid n \in \mathbf{N}\right\}$ and are constant (affine) there (cf. [12]). In this case, we write $\Lambda=\Lambda^{*}=\Lambda_{*}$.

Proposition 1 [12]. The normed space $X$ has (weakly) normal structure if and only if $X$ contains no (weakly convergent) limit-constant nonconstant sequence.

The normed space $X$ is said to have the (weak) sum-property (cf. [12]) if it contains no (weakly convergent) nonconstant limit-affine sequence $\left(x_{n}\right)_{n \in \mathbf{N}}$ for which $\left(\Lambda\left(x_{n}\right)\right)_{n \in \mathbf{N}}$ is nondecreasing. The sum-property is introduced in [12] to solve (at least partially) the problem whether normal structure is preserved under finite direct sums. Here, a property $(\mathrm{P})$ is said to be preserved under finite direct sums if, given a finite dimensional normed space $Z$ having a basis $\left(e_{i}\right)_{i \leqslant N}$ with

$$
\left\|\sum_{i=1}^{N} \zeta_{i} e_{i}\right\|_{Z} \leqslant\left\|\sum_{i=1}^{N} \zeta_{i}^{\prime} e_{i}\right\|_{Z} \quad \text { whenever } \zeta_{i}^{\prime} \geqslant \zeta_{i} \geqslant 0 \text { for all } i \leqslant N
$$

and given normed space $X_{i}, i \leqslant N$, with (P), then $\prod_{i=1}^{N} X_{i}$ endowed with the norm $\left\|\left(x_{i}\right)_{i \leqslant N}\right\|=\left\|\sum_{i=1}^{N}\right\| x_{i}\left\|_{X_{i}} e_{i}\right\|_{Z}$ has $(\mathrm{P})$.

In [12], it is proved that the sum-property is preserved under finite direct sums. So, since the sum-property obviously implies normal structure, it is desirable to find spaces having the sum-property or even to show that normal structure is equivalent to the sum-property. The latter is an open problem and is shown to hold for a very large class of spaces (cf. [12]).

We will see that, among the Orlicz sequence spaces, weakly normal structure is equivalent to the weak sum-property and normal structure is equivalent to the sum-property.

The idea of the following sufficient condition for weakly normal structure of normed spaces with a basis is due to Gossez and Lami-Dozo [7].

Proposition 2 [12]. Let $X$ have a basis $\left(e_{i}\right)_{i \in \mathrm{N}}$ which satisfies the condition

(GLD). There are $\varepsilon<1$ and $r>0$ such that $\|x\|=\left\|\sum_{i=1}^{\infty} x(i) e_{i}\right\| \geqslant 1$ $+r$ whenever $\left\|\sum_{i=1}^{j} x(i) e_{i}\right\|=1$ and $\left\|\sum_{i=j+1}^{\infty} x(i) e_{i}\right\| \geqslant \varepsilon$ for some $j \in \mathbf{N}$.

Then, $X$ contains no weakly convergent limit-affine sequence with $\inf \left\{\Lambda\left(x_{n}\right) \mid n \in \mathbf{N}\right\}$ $>0$. Consequently, $X$ has weakly normal structure, it even has the weak sum-property.

A sequence $\left(x_{n}\right)_{n \in \mathrm{N}}$ in $X$ is said to be almost convergent to some $x \in X$ if $\Lambda^{*}(x)<\Lambda_{*}(y)$ for all $y \neq x($ cf. $[4,9])$. This notion is useful in fixed point theory of nonexpansive mappings: If $T$ is a nonexpansive mapping and $\left(x_{n}\right)_{n \in \mathrm{N}}$ is a sequence with $\lim _{n \rightarrow \infty}\left\|x_{n}-T x_{n}\right\|=0$ which is almost convergent to some $x$, then $x$ is a fixed point of $T$.

2. Orlicz sequence spaces. Let $\varphi$ be a convex Orlicz function, i.e., $\varphi: \mathbf{R}_{+} \rightarrow \overline{\mathbf{R}}_{+}=$ $\mathbf{R}_{+} \cup\{+\infty\}$ is nondecreasing, nonconstant, convex and continuous at 0 with $\varphi(0)=0$. We set

$$
D(\varphi)=\{t>0 \mid \sup \{\varphi(s) \mid 0<s<t\}<\infty\} .
$$


Every Orlicz function $\varphi$ is continuous on the interior of $D(\varphi)$ and possesses a right derivative $\varphi^{\prime}(t)=\lim _{h \downarrow 0}\{(\varphi(t+h)-\varphi(t)) / h\}$ there.

Throughout the paper, let $\mathbf{K}$ be the scalar field $\mathbf{R}$ or $\mathbf{C}$. We define the functional $S_{\varphi}: \mathbf{K}^{\mathbf{N}} \rightarrow \overline{\mathbf{R}}_{+}$by

$$
S_{\varphi}(x)=\sum_{i=1}^{\infty} \varphi(|x(i)|) .
$$

The Orlicz sequence space $l_{\varphi}=l_{\varphi}(\mathbf{K})$ is defined by

$$
l_{\varphi}=\left\{x: \mathbf{N} \rightarrow \mathbf{K} \mid S_{\varphi}\left(c^{-1} x\right)<\infty \text { for some } c>0\right\} .
$$

Endowed with the norm

$$
\|x\|_{\varphi}=\inf \left\{c>0 \mid S_{\varphi}\left(c^{-1} x\right) \leqslant 1\right\}
$$

$l_{\varphi}$ is a real or complex Banach space according to $\mathbf{K}=\mathbf{R}$ or $\mathbf{K}=\mathbf{C}$. As usual, we write $l_{p}=l_{\varphi_{p}}$ and \|\|$_{p}=\|\|_{\varphi_{p}}$ for $\varphi_{p}(t)=t^{p}, 1 \leqslant p<\infty$, and

$$
\varphi_{p}(t)=\left\{\begin{array}{ll}
0, & t \leqslant 1 \\
\infty, & t>1
\end{array}\right\}, \quad p=\infty
$$

The unit vectors in $l_{\varphi}$ are denoted by $\left(e_{i}\right)_{i \in \mathbf{N}}$, where

$$
e_{i}(j)=\left\{\begin{array}{ll}
0, & i \neq j \\
1, & i=j
\end{array}\right\} .
$$

Whether $\left(e_{i}\right)_{i \in \mathbf{N}}$ is a basis of $l_{\varphi}$ depends strongly on the so-called $\Delta_{2}$-condition. The Orlicz function $\varphi$ is said to satisfy the $\Delta_{\alpha}$-condition at $0, \alpha>1$, if it is nondegenerate -i.e., if $\varphi(t)>0$ for all $t>0$ - and if there is $t>0$ with $\alpha t \in D(\varphi)$ and

$$
\beta(\alpha, t):=\sup \{\varphi(\alpha s) / \varphi(s) \mid 0<s<t\}<\infty .
$$

Obviously, $\beta(\alpha, t)<\infty$ whenever $\alpha t \in D(\varphi)$ and $\varphi$ satisfies the $\Delta_{\alpha}$-condition at 0 . It is important in our results that $l_{\varphi} \subset c_{0}$ if $\varphi$ is nondegenerate. The following fact is well known (cf. [13]):

Proposition 3. The following are equivalent.

(a) $\varphi$ satisifes the $\Delta_{\alpha}$-condition at 0 for all $\alpha>1$.

(b) $\varphi$ satisifes the $\Delta_{\alpha}$-condition at 0 for all some $\alpha>1$.

(c) $\varphi$ satisifes the $\Delta_{2}$-condition at 0 .

(d) $\lim \sup _{t \downarrow 0} t \varphi^{\prime}(t) / \varphi(t)<\infty$.

(e) $\left(e_{i}\right)_{i \in \mathrm{N}}$ is a boundedly complete basis of $l_{\varphi}$.

(f) $l_{\varphi}$ has no subspace isomorphic to $l_{\infty}$.

Although Lindenstrauss and Tzafriri only deal with real valued $\varphi$ in [13], the proof of Proposition 3 also works in our case.

We need some elementary properties of Orlicz functions and Orlicz sequence spaces.

Proposition 4. (i) If $\varphi$ satisfies the $\Delta_{2}$-condition at 0 , then

$$
\lim _{\alpha \downarrow 1} \beta\left(\alpha, \alpha^{-1} t\right)=1 \text { for all } t \in D(\varphi) .
$$


(ii) If $\varphi$ does not satisfy the $\Delta_{2}$-condition at 0 , then, for all $t>0$ and $\beta<\infty$, there is $s \in(0, t]$ with

$$
\infty>\varphi(\alpha s) \geqslant(\alpha-1) \beta \varphi(s) \text { for all } \alpha \in(1,2] .
$$

Proof. (i) Assume that $\varphi$ satisfies the $\Delta_{2}$-condition at 0 . Let $t \in D(\varphi)$ and $\beta>1$ be given. We have to find $\alpha>1$ such that $\beta\left(\alpha, \alpha^{-1} t\right) \leqslant \beta$. Since $t \in D(\varphi)$, there is $\tilde{\alpha}>1$ such that $\varphi(s) \leqslant \beta \varphi\left(\tilde{\alpha}^{-1} t\right)$ for all $s<t$. Set $\gamma=\beta\left(\tilde{\alpha}, \tilde{\alpha}^{-1} t\right)$ and choose $\alpha$ $\in(1, \tilde{\alpha}]$ with $\gamma(\alpha-1)+\tilde{\alpha}-\alpha \leqslant \beta(\tilde{\alpha}-1)$. For $s \in\left[\tilde{\alpha}^{-1} t, \alpha^{-1} t\right)$, we have $\varphi(\alpha s)$ $\leqslant \beta \varphi\left(\tilde{\alpha}^{-1} t\right) \leqslant \beta \varphi(s)$. Convexity of $\varphi$ implies for $s \in\left(0, \tilde{\alpha}^{-1} t\right)$ :

$$
\varphi(\alpha s) \leqslant \frac{\alpha-1}{\tilde{\alpha}-1} \varphi(\tilde{\alpha} s)+\frac{\tilde{\alpha}-\alpha}{\tilde{\alpha}-1} \varphi(s) \leqslant\left(\gamma \frac{\alpha-1}{\tilde{\alpha}-1}+\frac{\tilde{\alpha}-\alpha}{\tilde{\alpha}-1}\right) \varphi(s) \leqslant \beta \varphi(s) .
$$

(ii) Assume that $\varphi$ does not satisfy the $\Delta_{2}$-condition at 0 . Let $t>0$ and $\beta<\infty$ be given. Choose $\tilde{t} \leqslant t$ with $\varphi(2 \tilde{t})<\infty$. By Proposition $3(\mathrm{~d})$, there is $s \in(0, \tilde{t}]$ with $s \varphi^{\prime}(s) / \varphi(s) \geqslant \beta$. Fix $\alpha \in(1,2]$. Since $(\varphi(\alpha s)-\varphi(s)) /((\alpha-1) s) \geqslant \varphi^{\prime}(s)$ we finally obtain $\varphi(\alpha s) / \varphi(s) \geqslant 1+(\alpha-1) s \varphi^{\prime}(s) / \varphi(s) \geqslant(\alpha-1) \beta$.

The behaviour of $\varphi$ on $\varphi^{-1}([0,1])$ is important for the norm of $l_{\varphi}$. We define

$$
t(\varphi)=\sup \{t \mid \varphi(t) \leqslant 1\}, \quad C(\varphi)=\sup \{\varphi(t) \mid t<t(\varphi)\} .
$$

Proposition 5. If $\varphi$ satisfies the $\Delta_{2}$-condition at 0 , then $\lim _{\lambda \uparrow 1} S_{\varphi}(\lambda x) \geqslant C(\varphi)$ uniformly on $\left\{x \in l_{\varphi} \mid\|x\|_{\varphi}=1\right\}$.

Proof. Assume the contrary. Then there is $C<C(\varphi)$ such that, for all $\lambda<1$, there is $x_{\lambda}$ with $\left\|x_{\lambda}\right\|_{\varphi}=1$ and $S_{\varphi}\left(\lambda x_{\lambda}\right)<C$. Since $C<C(\varphi)$, there is $t>0$ with $\varphi(t)=C$ and $\tilde{\alpha}>1$ such that $\tilde{\alpha} t \in D(\varphi)$. Choose $\alpha \in(1, \tilde{\alpha}]$ with $\beta(\alpha, t) \leqslant C^{-1}$ and set $\lambda=\alpha^{-1 / 2}$. Since $S_{\varphi}\left(\lambda x_{\lambda}\right)<C$, we have $\lambda\left\|x_{\lambda}\right\|_{\infty}<t$. So, $S_{\varphi}\left(\sqrt{\alpha} x_{\lambda}\right)=$ $S_{\varphi}\left(\alpha \lambda x_{\lambda}\right) \leqslant C^{-1} S_{\varphi}\left(\lambda x_{\lambda}\right)<1$ which contradicts $\left\|x_{\lambda}\right\|_{\varphi}=1$.

The following condition $\left(\mathrm{G}_{\varepsilon}\right)$ defined for $\varepsilon \in(0,1)$ is closely related to the (GLD)-condition and to another condition (cf. [10]) which implies that any bounded sequence in $l_{\varphi}$ has a subsequence which is pointwise and almost convergent.

$\left(\mathrm{G}_{\varepsilon}\right)$ There exists $r>0$ such that $\|x+y\|_{\varphi} \geqslant 1+r$ whenever $\|x\|_{\varphi}=1,\|y\|_{\varphi} \geqslant \varepsilon$ and $x, y$ have disjoint supports.

The validity of $\left(\mathrm{G}_{\varepsilon}\right)$ for appropriate $\varepsilon$ depends on the number $C(\varphi)$ :

Proposition 6. Let $\varphi$ satisfy the $\Delta_{2}$-condition at 0 .

(a) Condition $\left(\mathrm{G}_{\varepsilon}\right)$ holds for some $\varepsilon \in(0,1)$ if and only if $C(\varphi)>\frac{1}{2}$.

(b) Condition $\left(\mathrm{G}_{\varepsilon}\right)$ holds for all $\varepsilon \in(0,1)$ if and only if $C(\varphi)=1$.

Proof. Assume $C=\max \left\{\frac{1}{2}, C(\varphi)\right\}<1$. Set $\varepsilon=C^{-1}-1$. Then $\varepsilon=1$ if $C=\frac{1}{2}$. We have $\|x\|_{\varphi}=t(\varphi)^{-1}$ whenever $\|x\|_{1} \leqslant C^{-1}$ and $|x(j)|=1$ for some $j \in \mathbf{N}$. Indeed, $S_{\varphi}(t x) \geqslant \varphi(t|x(j)|)=\varphi(t)>1$ if $t>t(\varphi)$, and, since $\sum_{i \neq j}|x(i)| \leqslant \varepsilon \leqslant 1$, we have $S_{\varphi}(t x) \leqslant \varphi(t)+\varepsilon \varphi(t)=C^{-1} \varphi(t)<1$ if $t<t(\varphi)$. So $\left\|t(\varphi) e_{1}\right\|_{\varphi}=1$, $\left\|\varepsilon t(\varphi) e_{2}\right\|_{\varphi}=\varepsilon$ and $\left\|t(\varphi) e_{1}+\varepsilon t(\varphi) e_{2}\right\|_{\varphi}=1$. This proves sufficiency in (a) and (b).

Assume $C(\varphi)>\frac{1}{2}$ in case (a) and $C(\varphi)=1$ in case (b). In case (b), let $\varepsilon>0$ be given. For necessity in (a) and (b), it suffices to find $\lambda<1$ such that $S_{\varphi}(\lambda x)+$ $S_{\varphi}(\lambda y)>1$ whenever $\|x\|_{\varphi}=1$ and $\|y\|_{\varphi}=\varepsilon$, where, in case $(a), \varepsilon \in(0,1)$ has to be chosen in an appropriate manner. 
In case (a), we choose $\lambda<1$ and $C>\frac{1}{2}$ such that $S_{\varphi}(\lambda z)>C$ whenever $\|z\|_{\varphi}=1$, and pick $\varepsilon \in(0,1)$ with $\gamma=\beta\left(\varepsilon^{-1}, \varepsilon t(\varphi)\right)<2 C$; we have $C\left(1+\gamma^{-1}\right)>1$.

In case (b), we put $\gamma=\beta\left(\varepsilon^{-1}, \varepsilon t(\varphi)\right)$, pick $C<1$ with $C\left(1+\gamma^{-1}\right)>1$ and choose $\lambda<1$ such that $S_{\varphi}(\lambda z)>C$ whenever $\|z\|_{\varphi}=1$.

Let $x, y$ be given with $\|x\|_{\varphi}=1,\|y\|_{\varphi}=\varepsilon$. Since $S_{\varphi}\left(\lambda \varepsilon^{-1} y\right)<1$, we have $\lambda\|y\|_{\infty}$ $<\varepsilon t(\varphi)$, so, $S_{\varphi}\left(\lambda \varepsilon^{-1} y\right) \leqslant \gamma S_{\varphi}(\lambda y)$. Hence $S_{\varphi}(\lambda x)+S_{\varphi}(\lambda y)>C+\gamma^{-1} S_{\varphi}\left(\lambda \varepsilon^{-1} y\right)$ $\geqslant C\left(1+\gamma^{-1}\right)>1$.

REMARK 1. In the first part of the proof we have shown in fact that $\left(e_{n}\right)_{n \in \mathrm{N}}$ is limit-constant if $C(\varphi) \leqslant \frac{1}{2}$, and that no subsequence of $\left(e_{n}\right)_{n \in \mathrm{N}}$ is almost convergent to its pointwise lilmit 0 if $C(\varphi)<1$. Moreover, in this part of the proof, we did not use the $\Delta_{2}$-condition.

The Orliz function $\varphi$ is said to be linear at 0 if there are $t>0$ and $c>0$ such that $\varphi(s)=c s$ for all $s \leqslant t$.

Proposition 7. If $\varphi$ is linear at 0 , then $l_{\varphi}$ has a subspace isometric to $l_{1}$.

Proof. Assume that $\varphi(s)=c s, s \leqslant t$, with $t>0, c>0$. Choose $m \in \mathbf{N}$ and $s \leqslant t$ with $m c s=1$. Set $x_{n}=s \sum_{i=1}^{m} e_{m n+i}$. Fix any finite sequence $\left(a_{k}\right)_{k \leqslant n}$ of scalars and set $a=\sum_{k=1}^{n}\left|a_{k}\right|$. Then $S_{\varphi}\left(a^{-1} \sum_{k=1}^{n} a_{k} x_{k}\right)=\sum_{k=1}^{n} m c a^{-1}\left|a_{k}\right| s=1$, i.e., $\left\|\sum_{k=1}^{n} a_{k} x_{k}\right\|_{\varphi}$ $=a=\sum_{k=1}^{n}\left|a_{k}\right|$.

3. Main results. All results in this section are valid for $\mathbf{K}=\mathbf{R}$ as well as for $K=\mathbf{C}$. Only in the proof of Theorem 1(d) do we have to distinguish between the cases $\mathbf{K}=\mathbf{R}$ and $\mathbf{K}=\mathbf{C}$.

Some of the proofs are very technical and involve a lot of estimations. So, we give these proofs in the last three sections.

We first characterize those Orlicz sequence spaces which contain an isometric copy of $l_{1}$.

THEOREM 1. The Orlicz sequence space $l_{\varphi}$ has a subspace isometric to $l_{1}$ if and only if $\varphi$ does not satisfy the $\Delta_{2}$-condition at 0 or $\varphi$ is linear at 0 .

Proof. See $§ 4$.

It is well known that $l_{\infty}$ contains an isometric copy of $l_{1}$. Theorem 1 tells us that $l_{\varphi}$ contains an isometric copy of $l_{1}$ whenever $l_{\varphi}$ has a subspace isomorphic-not necessarily isometric-to $l_{\infty}$. This result depends on the special structure of Orlicz sequence spaces because there are normed spaces isomorphic to $l_{\infty}$ which have normal structure (cf. [12]) and, consequently, cannot have a subspace isometric to $l_{1}$. On the other hand, if a normed space has a subspace isomorphic to $l_{\infty}$, then, for all $\varepsilon>0$, it has a subspace whose Banach-Mazur distance from $l_{1}$ is not bigger than $1+\varepsilon$. Theorem 1 shows that, among Orlicz sequence spaces, this holds even for $\varepsilon=0$.

We now show that the only Orlicz sequence spaces with the Schur property - weakly convergent sequences are norm convergent-are those isomorphic to $l_{1}$. 
THEOREM 2. The following are equivalent.

(a) $l_{\varphi}$ is isomorphic to $l_{1}$.

(b) $l_{\varphi}$ is Schur.

(c) $l_{\varphi}$ has weakly normal structure with respect to every equivalent norm.

(d) $\left(e_{n}\right)_{n \in \mathrm{N}}$ does not converge weakly to 0 .

(e) $\varphi^{\prime}(0)>0$.

Proof. (a) $\Rightarrow$ (b): $l_{1}$ is Schur. (b) $\Leftrightarrow$ (c): cf. [11]. (b) $\Rightarrow(d)$ : Trivial. (d) $\Rightarrow$ (e): Assume $\varphi^{\prime}(0)=0$. Choose a real valued Orlicz function $\tilde{\varphi}$ with $\lim _{t \rightarrow \infty} \tilde{\varphi}^{\prime}(t)=\infty$ such that $\varphi=\tilde{\varphi}$ in some neighbourhood of 0 . Then $\tilde{\varphi}^{\prime}(0)=0$ and the conjugate Orlicz function $\tilde{\varphi}^{*}(u)=\sup _{t \geqslant 0}\{t u-\tilde{\varphi}(t)\}$ is nondegenerate, i.e. $l_{\tilde{\varphi}^{*}} \subset c_{0}$. Since the identity is an isomorphism of $l_{\varphi}$ onto $l_{\tilde{\varphi}}$ and $l_{\varphi}^{*} \approx l_{\tilde{\varphi}^{*}}$ (cf. [13]), $l_{\varphi}^{*}$ can be identified with $l_{\tilde{\varphi}^{*}}$ endowed with some equivalent norm. So, $\left\langle x^{*}, e_{n}\right\rangle=x^{*}(n) \rightarrow 0, n \rightarrow \infty$, for all $x^{*} \in l_{\varphi}^{*}$. (e) $\Rightarrow(\mathrm{a})$ : If $\varphi^{\prime}(0)=c>0$, then $\varphi(t) \geqslant c t$ for all $t>0$. So, the identity is an isomorphism of $l_{\varphi}$ onto $l_{1}$ (cf. [13]).

In the next theorem, we give a characterization of Orlicz sequence spaces having normal structure.

THEOREM 3. The following are equivalent.

(a) $l_{\varphi}$ has normal structure.

(b) $l_{\varphi}$ has the sum-property.

(c) $\varphi$ satisfies the $\Delta_{2}$-condition at $0, \varphi$ is not linear at 0 and $C(\varphi)>\frac{1}{2}$.

(d) $l_{\varphi}$ has no subspace isometric to $l_{1}$ and $C(\varphi)>\frac{1}{2}$.

Proof. (b) $\Rightarrow$ (a): Trivial. (c) $\Leftrightarrow$ (d): Theorem 1. (a) $\Rightarrow$ (d): If $l_{\varphi}$ has a subspace isometric to $l_{1}$ then $l_{\varphi}$ cannot have normal structure. If $C(\varphi) \leqslant \frac{1}{2}$, then, according to Remark $1,\left(e_{n}\right)_{n \in \mathrm{N}}$ is limit-constant; thus, $l_{\varphi}$ does not have normal structure. (c) $\Rightarrow$ (b): See $\$$.

COROLlaRY 1. If $\varphi$ is real valued or if $\frac{1}{2}<\varphi(t)<\infty$ for at least one $t$, then $l_{\varphi}$ has normal structure if and only if $l_{\varphi}$ has no subspace isometric to $l_{1}$.

COROLlaRy 2. A reflexive Orlicz sequence space $l_{\varphi}$ has normal structure if and only if $C(\varphi)>\frac{1}{2}$.

EXAMPLE. Let $\varphi$ be an arbitrary real valued Orlicz function. For $\lambda>0$ set $\left.\varphi\right|_{\lambda}(t)=\varphi(t)$ if $t \leqslant \lambda^{-1}$ and $\left.\varphi\right|_{\lambda}(t)=\infty$ if $t>\lambda^{-1}$. Then the identity is an isomorphism of $l_{\varphi}$ onto $l_{\left.\varphi\right|_{\lambda}}$ and $\|x\|_{\left.\varphi\right|_{\lambda}}=\max \left\{\|x\|_{\varphi}, \lambda\|x\|_{\infty}\right\}$. Since $\varphi$ and $\left.\varphi\right|_{\lambda}$ coincide in a neighborhood of 0 and $C\left(\left.\varphi\right|_{\lambda}\right)=\min \left\{\varphi\left(\lambda^{-1}\right), 1\right\}$, Theorem 3 yields

COROLlaRY 3. $l_{\left.\varphi\right|_{\lambda}}$ has normal structure if and only if $l_{\varphi}$ has normal structure and $\varphi\left(\lambda^{-1}\right)>\frac{1}{2}$.

Let $l_{p, \lambda}$ be the space $l_{p}, 1 \leqslant p<\infty$, with norm $\|x\|_{p, \lambda}=\max \left\{\|x\|_{p}, \lambda\|x\|_{\infty}\right\}$. Since $l_{p, \lambda}=l_{\varphi_{p, \lambda}}$ and $\varphi_{p}(1 / \lambda)>\frac{1}{2}$ if and only if $\lambda<2^{1 / p}$, we obtain

COROLlaRY 4. $l_{p, \lambda}$ has normal structure if and only if $\lambda<2^{1 / p}$ and $p>1$.

The case $p=2$ has been investigated directly in [1]. 
Theorem 3 shows that, among the Orlicz sequence spaces, normal structure and the sum-property are equivalent. The same holds for the related weak properties as Theorem 4 shows.

THEOREM 4. The following are equivalent.

(a) $l_{\varphi}$ has weakly normal structure.

(b) $l_{\varphi}$ has the weak sum-property.

(c) $\varphi$ satisfies the $\Delta_{2}$-condition at 0 and $C(\varphi)>\frac{1}{2}$ or $\varphi^{\prime}(0)>0$.

Proof. (b) $\Rightarrow$ (a): Trivial. (c) $\Rightarrow$ (b): If $\varphi^{\prime}(0)>0$, then, by Theorem 2, every weakly convergent sequence converges in norm, i.e., $\lim _{n \rightarrow \infty} \Lambda\left(x_{n}\right)=0$. So, $l_{\varphi}$ has the weak sum-property. If $\varphi$ satisfies the $\Delta_{2}$-condition at 0 and $C(\varphi)>\frac{1}{2}$, then, by Proposition 6, $\left(\mathrm{G}_{\varepsilon}\right)$ holds for some $\varepsilon \in(0,1)$ and, hence, the basis $\left(e_{i}\right)_{i \in \mathrm{N}}$ of $l_{\varphi}$ satisfies (GLD). According to Proposition $2, l_{\varphi}$ has the weak sum-property.

(a) $\Rightarrow$ (c): Assume that $\varphi^{\prime}(0)=0, \varphi$ satisfies the $\Delta_{2}$-condition at 0 and $C(\varphi) \leqslant \frac{1}{2}$. Then by Remark $1,\left(e_{n}\right)_{n \in \mathrm{N}}$ is limit-constant, and, by Theorem 2, $\left(e_{n}\right)_{n \in \mathrm{N}}$ converges weakly to 0 . So, $l_{\varphi}$ does not have weakly normal structure. It remains to show that $l_{\varphi}$ does not have weakly normal structure if $\varphi$ does not satisfy the $\Delta_{2}$-condition at 0 . This is done in $\S 5$.

COROLlaRY 5. If $\varphi$ is real valued or if $\frac{1}{2}<\varphi(t)<\infty$ for at least one $t$, then $l_{\varphi}$ has weakly normal structure if and only if either $l_{\varphi}$ has no subspace isomorphic to $l_{\infty}$ or $l_{\varphi}$ is isomorphic to $l_{1}$.

In [10, Theorem 1], Lami-Dozo shows that the condition

(LD) Every bounded sequence in $l_{\varphi}$ has a subsequence which is pointwise and almost convergent.

holds if $\varphi$ is convex and satisfies

(CV) For all $R>0$ the map $k_{R}:(0,1) \rightarrow \mathbf{R}_{+}$defined by $k_{R}(\gamma)=$ $\inf _{0<t<R} \varphi(\gamma t) / \varphi(t)$ satisfies $0<k_{R}(\gamma)<1$ and $\lim _{\gamma \uparrow 1} k_{R}(\gamma)=1$.

Clearly, (CV) is only well defined for real valued $\varphi$ and implies the $\Delta_{2}$-condition since $k_{R}(\gamma)=\left(\beta\left(\gamma^{-1}, \gamma R\right)\right)^{-1}$. In [10], (CV) is only used to prove the following condition:

(*) For all $\varepsilon \in\left(0, \frac{1}{2}\right)$, there is an $r>0$ such that $\|x\|_{\varphi} \geqslant 1+r$ whenever

$$
\left\|\sum_{i=N+1}^{\infty} x(i) e_{i}\right\|_{\varphi}=1 \text { and }\left\|\sum_{i=1}^{N} x(i) e_{i}\right\|_{\varphi}>\varepsilon \quad \text { for some } N \in \mathbf{N} \text {. }
$$

which in turn implies (LD) even if $l_{\varphi}$ is replaced by any normed space with boundedly complete basis $\left(e_{i}\right)_{i \in \mathrm{N}}$.

The proof of [10, Theorem 1] in fact only works for real valued $\varphi$. But for real valued $\varphi$, we even know from Proposition 4 that $(\mathrm{CV})$ is equivalent to the $\Delta_{2}$-condition. So, (LD) holds if $\varphi$ satisfies the $\Delta_{2}$-condition at 0 and is real valued. Moreover, 
in our general case, we even have a characterization of those Orlicz sequence spaces for which (LD) holds:

THEOREM 5. Every bounded sequence in $l_{\varphi}$ has a subsequence which is pointwise and almost convergent if and only if $\varphi$ satisfies the $\Delta_{2}$-condition at 0 and $C(\varphi)=1$.

Proof. Sufficiency. If $\varphi$ satisfies the $\Delta_{2}$-condition at 0 and $C(\varphi)=1$, then $\left(\mathrm{G}_{\varepsilon}\right)$ holds for all $\varepsilon \in(0,1)$. This implies (*) and then (LD).

Necessity. If $C(\varphi)<1$ and $\varphi$ satisfies the $\Delta_{2}$-condition at 0 , then, according to Remark $1,\left(e_{n}\right)_{n \in \mathrm{N}}$ has no subsequence which is almost convergent to its pointwise limit 0 . Thus, (LD) does not hold. If $\varphi$ does not satisfy the $\Delta_{2}$-condition at 0 , then $l_{\varphi}$ does not have weakly normal structure. Hence, there is a sequence $\left(x_{n}\right)_{n \in \mathbf{N}}$ in $l_{\varphi}$ which converges weakly to some $x \in l_{\varphi}$ such that $\Lambda(x)=\Lambda\left(x_{n}\right)$ for all $n \in \mathbf{N}$ (cf. [12]). So, (LD) cannot hold.

\section{Proof of Theorem 1.}

(4.1) Necessity. Let $\varphi$ satisfy the $\Delta_{2}$-condition at 0 and be not linear at 0 . It is enough to show that the following property holds:

(C) If $\left\|x_{n}\right\|_{\varphi}=1$ and $x_{n} \rightarrow 0$ pointwise, then $\left\|x_{1}+x_{n}\right\|_{\varphi}<2$ for some $n>1$.

Indeed, if $\left\|\sum_{k=1}^{n} a_{k} y_{k}\right\|_{\varphi}=\sum_{k=1}^{n}\left|a_{k}\right|$ for every finite sequence $\left(a_{k}\right)_{k \leqslant n}$ of scalars, then we may choose a subsequence $\left(z_{n}\right)_{n \in \mathrm{N}}$ of $\left(y_{n}\right)_{n \in \mathrm{N}}$ which converges pointwise and put $x_{n}=\frac{1}{2}\left(z_{2 n+1}-z_{2 n}\right)$. Then, clearly, $x_{n} \rightarrow 0$ pointwise, $\left\|x_{n}\right\|_{\varphi}=1$ and $\left\|x_{1}+x_{n}\right\|_{\varphi}=2$ for all $n \geqslant 1$.

We show (C). Assume $\left\|x_{n}\right\|_{\varphi}=1$ and $x_{n} \rightarrow 0$ pointwise. Fix any $\gamma \in(1,2)$. Pick $\tilde{i} \in \mathbf{N}$ such that $\left|x_{1}(i)\right|<(\gamma-1) t(\varphi)$ for all $i>\tilde{i}$. Choose $N \in \mathbf{N}$ such that $\left|x_{n}(i)\right|$ $<(\gamma-1) t(\varphi)$ for all $i \leqslant \tilde{i}$ and $n>N$. We have (i) $\left\|u_{n}\right\|_{\infty}<\gamma t(\varphi) / 2$ for all $n>N$, $u_{n}:=\frac{1}{2}\left(x_{1}+x_{n}\right)$. Choose $j \in \mathbf{N}$ such that $t=\left|x_{1}(j)\right|>0$. Then, $t \leqslant t(\varphi)$ and $0<\mu=\lim _{s \uparrow t} \varphi(s) \leqslant 1$. Since $\varphi$ is not linear at 0 , there are $c>1$ and $v>\frac{1}{2} t$ such that $\varphi(c v)=\frac{1}{2} \mu$. Since $\lim _{n \rightarrow \infty}\left|u_{n}(j)\right|=t / 2$, there is $n>N$ such that $\left|u_{n}(j)\right|<v$. So, $\varphi\left(\alpha\left|u_{n}(j)\right|\right)<\alpha c^{-1} \mu / 2$ for all $\alpha \in(1, c]$. If $1<\alpha<\min \{\sqrt{2 / \gamma}, c\}$ then

$$
\begin{aligned}
& S_{\varphi}\left(\alpha u_{n}\right)<\beta\left(\alpha^{2}, \frac{\gamma}{2} t(\varphi)\right) \sum_{i \neq j} \varphi\left(\alpha^{-1}\left|u_{n}(i)\right|\right)+\alpha c^{-1} \mu / 2 \\
& \quad \leqslant \beta\left(\alpha^{2}, \frac{\gamma}{2} t(\varphi)\right) \sum_{i \neq j} \frac{1}{2}\left\{\varphi\left(\alpha^{-1}\left|x_{1}(i)\right|\right)+\varphi\left(\alpha^{-1}\left|x_{n}(i)\right|\right)\right\}+\alpha c^{-1} \mu / 2 \\
& \quad<\beta\left(\alpha^{2}, \frac{\gamma}{2} t(\varphi)\right)\left(1-\frac{1}{2} \varphi\left(\alpha^{-1} t\right)\right)+\alpha c^{-1} \mu / 2 \rightarrow 1-\left(1-c^{-1}\right) \mu / 2<1 \quad \text { if } \alpha \downarrow 1 .
\end{aligned}
$$

Hence, $S_{\varphi}\left(\alpha u_{n}\right)<1$ for some $\alpha>1$ and, consequently, $\left\|x_{1}+x_{n}\right\|_{\varphi} \leqslant 2 \alpha^{-1}<2$.

(4.2) Sufficiency. According to Proposition 7, it remains to show that $l_{\varphi}$ has a subspace isometric to $l_{1}$ if $\varphi$ does not satisfy the $\Delta_{2}$-condition at 0 . So, assume that $\varphi$ does not satisfy the $\Delta_{2}$-condition at 0 . It is enough to find a sequence $\left(x_{n}\right)_{n \in \mathrm{N}}$ in $l_{\varphi}$ such that $\left\|x_{n}\right\|_{\varphi} \leqslant 1$ for all $n \in \mathbf{N}$ and $\left\|\sum_{k=1}^{n} a_{k} x_{k}\right\|_{\varphi} \geqslant \sum_{k=1}^{n}\left|a_{k}\right|$ for every finite sequence $\left(a_{k}\right)_{k \leqslant n}$ of scalars.

Assume first that there are sequences $\left(t_{m}\right)_{m \in \mathbf{N}} \subset \mathbf{R}_{+}$and $\left(k_{m}\right)_{m \in \mathbf{N}} \subset \mathbf{N}$ such that

$$
\begin{gathered}
k_{m} \varphi\left(t_{m}\right) \leqslant 2^{-m}, \\
\liminf _{m \rightarrow \infty} k_{m} \varphi\left(\alpha t_{m}\right)>0 \text { for all } \alpha>1 .
\end{gathered}
$$


Set $s_{m}=\sum_{j=1}^{m-1} k_{j}$ and $x_{n}\left(s_{m}+k\right)=\sigma_{n, m} t_{m}, 1 \leqslant k \leqslant k_{m}$, where $\sigma_{n, m}$ are certain scalars with $\left|\sigma_{n, m}\right|=1$ (to be defined below).

No matter how the $\sigma_{n, m}$ are defined, we have by (1) for all $n \in \mathbf{N}$ :

$$
S_{\varphi}\left(x_{n}\right)=\sum_{m=1}^{\infty} k_{m} \varphi\left(t_{m}\right) \leqslant \sum_{m=1}^{\infty} 2^{-m}=1 \text {, }
$$

hence $\left\|x_{n}\right\|_{\varphi} \leqslant 1$.

We now define the $\sigma_{n, m}$. Set $r_{j}=j$ if $\mathbf{K}=\mathbf{C}$ and $r_{j}=2$ if $\mathbf{K}=\mathbf{R}, j \in \mathbf{N}$. Let, for all $p \in \mathbf{N}, \Omega_{p}=\left(\omega_{p, n, \nu}\right)$ be some $p \times r_{p}^{p}$ matrix whose columns are just all combinations of $p$-tuples of unit roots of order $r_{p}$. For example $\Omega_{2}=\left(\begin{array}{ccc}+1+1-1 & -1 \\ +1-1 & -1 & -1\end{array}\right)$. We define $\left(\sigma_{n, m}\right)=\left({ }^{\Omega_{1} \Omega_{2} \Omega_{3} \cdots}\right)$ i.e.,

$$
\sigma_{n, m}=\left\{\begin{array}{cc}
\omega_{p, n, \nu} & n \leqslant p \\
1 & n>p
\end{array}\right\}, \quad m=\sum_{j=1}^{p-1} r_{j}^{j}+\nu, \quad 1 \leqslant \nu \leqslant r_{p}^{p} .
$$

Let scalars $a_{j}, j \leqslant n$, be given with $\sum_{j=1}^{n}\left|a_{j}\right|=1$. We have to show that $\left\|\sum_{j=1}^{n} a_{j} x_{j}\right\|_{\varphi} \geqslant 1$. It is enough to show that $S_{\varphi}\left(\beta \sum_{j=1}^{n} a_{j} x_{j}\right)=\infty$ for all $\beta>1$. So, fix $\beta>1$. Pick $\gamma<1$ such that $\alpha=\beta \gamma>1$. Take $\tilde{p}>n$ such that, for every $p \geqslant \tilde{p}$ and $j \leqslant n$, there is a unit root $\zeta_{p, j}$ of order $r_{p}$ such that

$$
\operatorname{Re}\left(a_{j} \zeta_{p, j}\right) \geqslant \gamma\left|a_{j}\right|, j \leqslant n, p \geqslant \tilde{p}, \text { and, hence, }\left|\sum_{j=1}^{n} a_{j} \zeta_{p, j}\right| \geqslant \gamma, p \geqslant \tilde{p} \text {. }
$$

By definition of $\Omega_{p}$, there are numbers $m_{p}=\sum_{i=1}^{p-1} r_{i}^{i}+\nu_{p}, 1 \leqslant \nu_{p} \leqslant r_{p}^{p}$, such that

$$
\zeta_{p, j}=\omega_{p, j, \nu_{p}}=\sigma_{j, m_{p}} \text { for all } j \leqslant n, p \geqslant \tilde{p} .
$$

Using (3) and (4), we finally obtain

$$
S_{\varphi}\left(\beta \sum_{j=1}^{n} a_{j} x_{j}\right) \geqslant \sum_{p=\tilde{p}}^{\infty} k_{m_{p}} \varphi\left(\beta t_{m_{p}}\left|\sum_{j=1}^{n} a_{j} \zeta_{p, j}\right|\right) \geqslant \sum_{p=\tilde{p}}^{\infty} k_{m_{p}} \varphi\left(\alpha t_{m_{p}}\right)=\infty
$$

because, by (2), $\liminf _{p \rightarrow \infty} k_{m_{p}} \varphi\left(\alpha t_{m_{p}}\right)>0$.

It remains to construct the sequences $\left(t_{m}\right)_{m \in N}$ and $\left(k_{m}\right)_{m \in N}$ with (1) and (2). We distinguish two cases: (a) $\varphi$ is degenerate. Then $t=\sup \{s \geqslant 0 \mid \varphi(s)=0\}$. Set $k_{m}=1$ and choose an arbitrary sequence $\left(t_{m}\right)_{m \in \mathbf{N}}$ with $t_{m} \uparrow t$. Then, $k_{m} \varphi\left(t_{m}\right)=0<$ $2^{-m}$. Given $\alpha>1$, there are $u>t$ and $\tilde{m} \in \mathbf{N}$ such that $\alpha t_{m}>u$ for all $m>\tilde{m}$. Hence, $\liminf _{m \rightarrow \infty} k_{m} \varphi\left(\alpha t_{m}\right) \geqslant \varphi(u)>0$.

(b) $\varphi$ is nondegenerate. Then, there is a sequence $\left(t_{m}\right)_{m \in N}$ with $\varphi\left(t_{m}\right) \leqslant 2^{-m-1}$ and $\varphi\left(\alpha t_{m}\right) / \varphi\left(t_{m}\right) \geqslant(\alpha-1) 2^{m}$ for all $\alpha \in(1,2)$ (Proposition 4(ii)). Let $k_{m}$ be the largest integer with $2^{m} k_{m} \varphi\left(t_{m}\right) \leqslant 1$. For all $\alpha \in(1,2): k_{m} \varphi\left(\alpha t_{m}\right) \geqslant(\alpha-1) 2^{m} \varphi\left(t_{m}\right)$ $=(\alpha-1)\left(2^{m}\left(k_{m}+1\right) \varphi\left(t_{m}\right)-2^{m} \varphi\left(t_{m}\right)\right) \geqslant(\alpha-1) / 2>0$.

5. Remaining part of the proof of Theorem 3. We assume that $C(\varphi)>\frac{1}{2}, \varphi$ satisfies the $\Delta_{2}$-condition at 0 and $\varphi$ is not linear at 0 . Suppose that $l_{\varphi}$ does not have the sum-property. Then, there is a limit-affine sequence $\left(x_{n}\right)_{n \in \mathbf{N}}$ in $l_{\varphi}$ with $\inf _{n \in \mathbf{N}} \Lambda\left(x_{n}\right) \geqslant \varepsilon>0$. If $r>0, x \in l_{\varphi}$ and $\left(y_{n}\right)_{n \in \mathbf{N}}$ is a subsequence of $\left(x_{n}\right)_{n \in \mathbf{N}}$, 
then $\left(r\left(y_{n}-x\right)\right)_{n \in \mathbf{N}}$ is also limit-affine with $\inf _{n \in \mathbf{N}} \Lambda\left(r\left(y_{n}-x\right)\right) \geqslant r \varepsilon$. Since pointwise limits of bounded sequences in $l_{\varphi}$ belong to $l_{\varphi}$-the basis is boundedly complete-we may assume that $x_{n} \rightarrow 0$ pointwise and $\Lambda\left(x_{n}\right) \rightarrow 1$.

Since $C(\varphi)>\frac{1}{2}$, there is $\tilde{\beta}<1$ such that $2 \varphi(\tilde{\beta} t)>1, t=t(\varphi)$. We choose an arbitrary $\hat{\beta} \in(\tilde{\beta}, 1)$ and claim: There exists $N \in \mathbf{N}$ such that $\left\|x_{n}\right\|_{\infty} \leqslant \hat{\beta} t$ for all $n>N$.

We proof (1) indirectly. If (1) does not hold, then-since $x_{n} \rightarrow 0$ pointwise-for all $\tilde{i} \in \mathbf{N}$ and $N \in \mathbf{N}$, there are $i \geqslant \tilde{i}$ and $n>N$ such that $\left|x_{n}(i)\right|>\hat{\beta} t$. Pick $\alpha>1$ with $\alpha \tilde{\beta}<\hat{\beta}$ and put $\varepsilon=(\hat{\beta}-\alpha \tilde{\beta}) t>0$. Choose $M \in \mathbf{N}$ such that $\Lambda\left(x_{n}\right)<\alpha$ for all $n>M$. There are $k>M$ and $i \geqslant 1$ such that $\left|x_{k}(i)\right|>\hat{\beta} t$. Take $\tilde{i}>i$ such that $\left|x_{k}(j)\right|<\varepsilon$ for all $j \geqslant \tilde{i}$. Pick $N>k$ such that $\left|x_{n}(i)\right|<\varepsilon$ for all $n>N$ and (i): $\left\|x_{n}-x_{k}\right\|_{\varphi}<\alpha$ for all $n>N$. There are $n>N$ and $j \geqslant \tilde{i}$ such that $\left|x_{n}(j)\right|>\hat{\beta} t$. This all together yields $\left|x_{n}(i)-x_{k}(i)\right|>\hat{\beta} t-\varepsilon=\alpha \tilde{\beta} t,\left|x_{n}(j)-x_{k}(j)\right|>\hat{\beta} t-\varepsilon=$ $\alpha \tilde{\beta} t$. So, $S_{\varphi}\left(\alpha^{-1}\left(x_{n}-x_{k}\right)\right)>2 \varphi(\tilde{\beta} t)>1$ which contradicts (i) and completes the proof of (1).

Fix any $\beta \in(\hat{\beta}, 1)$ and set $\delta=(\beta-\hat{\beta}) t$. Using $\lim _{n \rightarrow \infty} x_{n}(i)=0, i \in \mathbf{N}$, and $\lim _{i \rightarrow \infty} x_{n}(i)=0, n \in \mathbf{N}$, we construct inductively a subsequence of $\left(x_{n}\right)_{n \in \mathbf{N}}$-again denoted by $\left(x_{n}\right)_{n \in \mathbf{N}}$-and an increasing sequence $\left(i_{n}\right)_{n \in \mathbf{N}}$ of indices such that

$$
\left|x_{n}(i)\right|<\delta \text { whenever } i \leqslant i_{n-1} \text { or } i \geqslant i_{n}, n \in \mathbf{N} .
$$

Using the triangle inequality pointwise, we obtain $\left\|x_{m}-x_{n}\right\|_{\infty}<\hat{\beta} t+\delta=\beta t$.

Since $\Lambda\left(x_{n}\right) \rightarrow 1$, there is a subsequence of $\left(x_{n}\right)_{n \in \mathbf{N}}$-again denoted by $\left(x_{n}\right)_{n \in \mathbf{N}}$ -and $\gamma<1$ such that $\left\|x_{m}-x_{n}\right\|_{\varphi}>\gamma^{-1} \beta$ for all $n \neq m$. Therefore

$$
\left\|x_{m}-x_{n}\right\|_{\infty}<\beta t<\gamma t\left\|x_{m}-x_{n}\right\|_{\varphi} \text { for all } m \neq n \text {. }
$$

Choose $j \in \mathbf{N}$ such that $s=\left|x_{1}(j)\right| / \Lambda\left(x_{1}\right)>0$. We have $s \leqslant \gamma t$. Set $\lambda=$ $\Lambda\left(x_{1}\right) /\left(1+\Lambda\left(x_{1}\right)\right)$. Since $\varphi$ is continuous on $[0, \gamma t]$ and not linear at 0 , there are $c>1$ and $u>\lambda s$ such that $\varphi(c u)=\lambda \varphi(s)$. Hence,

$\lim _{m \rightarrow \infty} \lim _{n \rightarrow \infty}\left|y_{n, m}(j)\right|=\lambda s, \quad y_{n, m}:=\left(2 x_{n}-x_{1}-x_{m}\right) /\left(\left\|x_{n}-x_{1}\right\|_{\varphi}+\left\|x_{n}-x_{m}\right\|_{\varphi}\right)$, implies

$$
\lim _{m \rightarrow \infty} \lim _{n \rightarrow \infty} \varphi\left(\alpha\left|y_{n, m}(j)\right|\right) \leqslant \alpha c^{-1} \lambda \varphi(s) \text { for all } \alpha \in(1, c) .
$$

For $\lambda_{n, m}=\left\|x_{n}-x_{1}\right\|_{\varphi} /\left(\left\|x_{n}-x_{1}\right\|_{\varphi}+\left\|x_{n}-x_{m}\right\|_{\varphi}\right), \quad z_{n, l}=\left(x_{n}-x_{l}\right) /\left\|x_{n}-x_{l}\right\|_{\varphi}$ we have

$$
\begin{gathered}
y_{n, m}=\lambda_{n, m} z_{n, 1}+\left(1-\lambda_{n, m}\right) z_{n, m}, \quad n>m>1, \\
\left\|z_{n, l}\right\|_{\infty}<\gamma t \text { and } S_{\varphi}\left(z_{n, l}\right) \leqslant 1, \quad n>l \geqslant 1 .
\end{gathered}
$$

Set $\tilde{\alpha}=\min \left(c, \gamma^{-1}\right)>1$. Application of (5) and (6) yields for $\alpha \in(1, \tilde{\alpha})$ :

$$
\begin{aligned}
S_{\varphi}\left(\alpha y_{n, m}\right) \leqslant & \beta(\alpha, \gamma t) \sum_{i \neq j}\left\{\lambda_{n, m} \varphi\left(\left|z_{n, 1}(i)\right|\right)+\left(1-\lambda_{n, m}\right) \varphi\left(\left|z_{n, m}(i)\right|\right)\right\} \\
& +\varphi\left(\alpha\left|y_{n, m}(j)\right|\right) \\
\leqslant & \beta(\alpha, \gamma t)\left\{1-\lambda_{n, m} \varphi\left(\left|z_{n, 1}(j)\right|\right)\right\}+\varphi\left(\alpha\left|y_{n, m}(u)\right|\right) \\
:= & h(\alpha, n, m) .
\end{aligned}
$$


Using (4), $\lim _{\alpha \downarrow 1} \beta(\alpha, \gamma t)=1, \lim _{m \rightarrow \infty} \lim _{n \rightarrow \infty} \lambda_{n, m}=\lambda$ and $\lim _{n \rightarrow \infty}\left|z_{n, 1}(j)\right|=$ $s$, we deduce $\lim _{a \downarrow l} \lim _{m \rightarrow \infty} \lim _{n \rightarrow \infty} h(\alpha, n, m)=1-\lambda\left(1-c^{-1}\right) \varphi(s)<1$.

So, by (7), there is $\alpha \in(1, \tilde{\alpha}), m>1$ and $N>m$ such that $S_{\varphi}\left(\alpha y_{n, m}\right) \leqslant$ $h(\alpha, n, m)<1$ for all $n>N$. From the very definition of $y_{n, m}$ we finally obtain $\left\|x_{n}-\frac{1}{2}\left(x_{1}+x_{m}\right)\right\|_{\varphi} \leqslant \alpha^{-1} \frac{1}{2}\left(\left\|x_{n}-x_{1}\right\|_{\varphi}+\left\|x_{n}-x_{m}\right\|_{\varphi}\right), n>N$. Letting $n \rightarrow \infty$, this contradicts the limit-affineness of $\left(x_{n}\right)_{n \in \mathrm{N}}$.

6. Remaining part of the proof of Theorem 4. We suppose that $\varphi$ does not satisfy the $\Delta_{2}$-condition at 0 . Set $t=\max \{s>0 \mid \varphi(s)=0\}$. We distinguish the two cases $t=0$ and $t>0$. In each case, we construct sequences $\left(t_{n}\right)_{n \in \mathbf{N}}$ and $\left(\alpha_{n}\right)_{n \in \mathbf{N}}$ in $\mathbf{R}_{+}$ and $\left(k_{n}\right)_{n \in \mathbf{N}}$ in $\mathbf{N}$ with $\alpha_{n} \downarrow 1, k_{n} \varphi\left(t_{n}\right)<2^{-n}$ and $k_{n} \varphi\left(\alpha_{n} t_{n}\right) \geqslant 1$.

Case 1. $t>0$. Define $\alpha_{n}>1$ by $\varphi\left(\alpha_{n} t\right)=(k n)^{-1}, k>C(\varphi)^{-1}$. Set $k_{n}=k n$ and $t_{n}=t$. Then $k_{n} \varphi\left(\alpha_{n} t_{n}\right)=1$ and $k_{n} \varphi\left(t_{n}\right)=0<2^{-n}$.

Case 2. $t=0$. Using Proposition 4(ii), we inductively construct a decreasing sequence $\left(t_{n}\right)_{n \in \mathrm{N}}$ of positive numbers with

(i) $\varphi\left(\alpha_{n} t_{n}\right)>2^{n+1} \varphi\left(t_{n}\right), \alpha_{n}:=1+n^{-1}, \varphi\left(2 t_{1}\right) \leqslant 1$.

Let $k_{n}$ be the least integer with $k_{n} \varphi\left(\alpha_{n} t_{n}\right) \geqslant 1$. We have

$$
k_{n} \varphi\left(\alpha_{n} t_{n}\right) \leqslant\left(k_{n}-1\right) \varphi\left(\alpha_{n} t_{n}\right)+\varphi\left(2 t_{1}\right)<1+1=2
$$

and, thus, $k_{n} \varphi\left(t_{n}\right)<2^{-n-1} k_{n} \varphi\left(\alpha_{n} t_{n}\right)<2^{-n}$.

Both cases. We set $s_{n}=\sum_{i=1}^{n-1} k_{i}$ and $x_{n}=t_{n} \sum_{i=1}^{k_{n}} e_{s_{n}+i} \in l_{\varphi}$. The $x_{n}$ have mutually disjoint supports. So, for arbitrary $\xi=\left(\xi_{n}\right)_{n \in \mathbf{N}} \in c_{0}$, the pointwise sum $T \xi=$ $\sum_{n=1}^{\infty} \xi_{n} x_{n}$ is well defined. Since

$$
S_{\varphi}\left(\|\xi\|_{\infty}^{-1} T \xi\right) \leqslant \sum_{n=1}^{\infty} k_{n} \varphi\left(t_{n}\right)<\sum_{n=1}^{\infty} 2^{-n}=1,
$$

$T: c_{0} \rightarrow l_{\varphi}$ is linear and bounded with $\|T\| \leqslant 1$. Obviously, $x_{n}=T \delta_{n}, \delta_{n}$ the $n$th unit vector of $c_{0}$. Since $\delta_{n} \rightarrow 0$ weakly in $c_{0}, x_{n} \rightarrow 0$ weakly in $l_{\varphi}$.

Let $x=\sum_{k=1}^{n} \lambda_{k} x_{k}, \lambda_{k} \geqslant 0, \sum_{k=1}^{n} \lambda_{k}=1$, be arbitrarily given. Then, $\left\|x_{n}-x\right\|_{\varphi} \leqslant$ $\left\|\delta_{n}-\sum_{k=1}^{n} \lambda_{k} \delta_{k}\right\|_{x}=1, n>m$, and so $\Lambda^{*}(x) \leqslant 1$.

Conversely, we have $\Lambda_{*}(x) \geqslant 1$ since $\left\|x_{n}-x\right\|_{\varphi} \geqslant \alpha_{n}^{-1}, n>m$, because of $S_{\varphi}\left(\alpha_{n}\left(x_{n}-x\right)\right) \geqslant k_{n} \varphi\left(\alpha_{n} t_{n}\right) \geqslant 1, n>m$.

Hence, $\Lambda^{*}(x)=\Lambda_{*}(x)=1$ and $\left(x_{n}\right)_{n \in \mathbf{N}}$ is limit-constant. Therefore, $l_{\varphi}$ does not have weakly normal structure.

ADDED IN PROOF. The author has shown the sum-property does not imply normal structure (cf. the text after Proposition 1) and that an Orlicz sequence space has a subspace isomorphic to $l_{\infty}$ if and only if it has a subspace isometric to $l_{\infty}$ (cf. the text after Theorem 1). These results will be published elsewhere.

\section{REFERENCES}

1. J. P. Baillon and R. Schöneberg, Asymptotic normal structure and fixed points of nonexpansiee maps, Proc. Amer. Math. Soc. 81 (1981), 257-264.

2. M. S. Brodskii and D. P. Mil'man, On the center of a convex set, Dokl. Akad. Nauk SSSR 59 (1948). 837-840. (Russian)

3. F. E. Browder, Nonlinear mappings of nonexpansive and accretive type in Banach spaces, Bull. Amer. Math. Soc. 73 (1967), 875-881.

4. M. Edelstein, The construction of an as!mptotic center with a fixed point propert!. Bull. Amer. Math. Soc. 78 (1972), 206-208. 
5. K. Goebel, On a fixed point theorem for multivalued nonexpansive mappings, Ann. Univ. Mariae Curie-Sklodowska Sect. A 29 (1975), 69-72.

6. D. Göhde, Zum Prinzip der kontraktiven Abbildung, Math. Nachr. 30 (1965), 251-258.

7. J. P. Gossez and E. Lami-Dozo, Structure normale et base de Schauder, Bull. Acad. Royale Belgique 15 (1969), 673-681.

8. W. A. Kirk, A fixed point theorem for mappings which do not increase distances, Amer. Math. Monthly 72 (1965), 1004-1006.

9. E. Lami-Dozo, Centres asymptotiques dans certains F-espaces, Boll. Un. Mat. Ital. B 17 (1980), 740-747.

10. ___ Asymptotic centers in particular spaces, Fixed Point Theory (Proc. Conf. Sherbrooke, 1980), Lecture Notes in Math., vol. 886, Springer-Verlag, Berlin and New York, 1981.

11. Th. Landes, A characterization of totally normal structure, Arch. Math. 37 (1981), 248-255.

12. __ Permanence properties of normal structure, Pacific J. Math. 110 (1984), 125-143.

13. J. Lindenstrauss and L. Tzafriri, Classical Banach spaces. I, Sequence spaces, Springer-Verlag, Berlin and New York, 1977.

Universität - GH PAderborn, Warburgerstrasse 100, D - 4790 Paderborn, Federal Republic OF GERMANY 